\title{
Research Paper \\ Comparison of Functional Balance and Probability of Falling in the Elderly with and Without a History of Falling Living in Care Centers
}

\author{
Seyyed Mojtaba Tabatabaias ${ }^{1}\left(\mathbb{0}\right.$, *Parisa Sedaghati $^{1}$ (1) Fariba Javazi $^{1}$
}

1. Department of Sport Injuries and Corrective Exercise, Faculty of Physical Education and Sport Sciences, University of Guilan, Rasht, Iran.

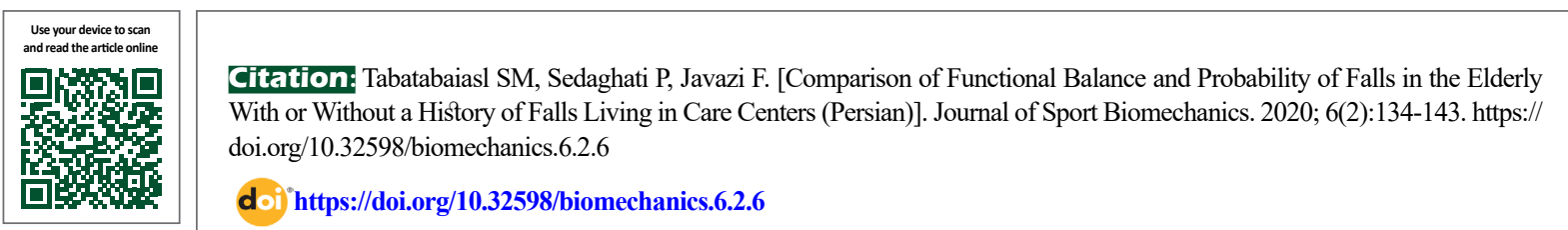

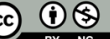

Article Info:

Received: 21 Jul 2020

Accepted: 09 Aug 2020

Available Online: 01 Sep 2020

Keywords:

Aged, Functional Balance, Probability of Fall, Fall History

\section{A B STRACT}

Objective The purpose of this study was to compare functional balance and probability of fall in elderly with or without history of fall in care centers.

Methods The statistical population of the present study consisted of 30 elderly men and women over 60 years of two elderly nursing centers in rasht. These specimens were selected according to the inclusion criteria and exit criteria. The age range was between 60 and 74 years. Individuals ( 15 people )were divided into two groups of aged persons (15 persons) and with a history of land (15 people). People who had no history of eating during the last six months have been knocked down in the group without a history of land eating and people who had tripped once or more in the last six months. the evaluation of the balance and the possibility of seniors fall with tests (leaves and time of rise and departure) were carried out. SPSS software was used to analyze the data. from the shayper-vink test to be natural for data distribution, descriptive statistics were used in the form of statistics such as mean, standard deviation, median, change range and direction of data describing and in the deductive statistics section with respect to comparing the variables from independent t-test.

Results Normality test results confirmed normality of data in the variables of performance equilibrium and probability of fall there was a significant difference between functional balance index in the elderly with a history of fall or without the history of fall. there was a meaningful difference between fall risk index in the elderly with a history of fall or without the history of fall.

Conclusion according to the results of this study, individuals with no history of fall in performance balance indices and probability of fall were better than those with history of fall.

\section{Extended Abstract}

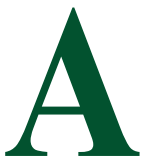

\section{Introduction}

ging affects all major body systems, including anatomical and functional designs, so the efficiency of postural and motor sys- tems, which are responsible for postural stability, gradually decreases with age [4]. In most cases, low balance is the leading cause of falls [5]. Balance is the ability to maintain body position within the level of reliance. During static and dynamic balance, body position is controlled by shifting the center of gravity and initiating appropriate responses to return the body to a stable position, a complex process

\section{* Corresponding Author:}

Parisa Sedaghati, PhD.

Address: Department of Sport Injuries and Corrective Exercise, Faculty of Physical Education and Sport Sciences, University of Guilan, Rasht, Iran. Tel: +98 (912) 2768326

E-mail: sedaghati@guilan.ac.ir 
in which the visual senses, somatosensory, vestibular, and musculoskeletal systems play an important role [6]. The low balance in the elderly is accompanied by an increase in the rate of falls; every year, one-third of the elderly with 65 years and older experience falls.

Falling in the elderly causes severe complications such as severe fractures, especially in the bones of the neck, wrist, pelvis, and soft tissue injuries [7]. Research has also shown that half of the elderly with a history of falls have limited mobility [8]. Although physical and mental deficiencies caused by old age can be partially eliminated with drug therapy methods, it seems that to deal with this significant and growing problem of human societies, safer and more appropriate solutions should be found [9].

\section{Methods}

The present study was a causal-comparative study that was conducted in the field. The study subjects included 30 elderly men and women over 60 years of age from two nursing homes in Rasht and were selected by convenience sampling. Individuals who had no history of falls during the last six months were in the group without a history of falls. Individuals who had fallen once or more during the previous six months [14] were in the group with a history of falls. Balance and probability of falls in the elderly were assessed by Berg test and TUG test [15]. The code of ethics was also received from the Research Institute of Medical Sciences Technology of Guilan University. SPSS statistical software was used for data analysis. The Shapiro-Wilk test was used for normal distribution of data; descriptive statistics tests were used in statistics such as Mean $\pm \mathrm{SD}$, median, and amplitude of changes and describe the inferential data statistics section to the comparison of variables, independent T-test was used.

\section{Results}

The demographic information of the elderly is given in Table 1. According to the results of the independent t-test, there was a significant difference between functional balance index in the elderly with a history of falls and no history of falls; the balance scores of the elderly without a history of falls were significantly higher than the elderly with a history of falls $(\mathrm{P}<0.007)$.

The elderly had a history of falls $(\mathrm{P}<0.007)$. According to the results of the independent t-test, there was a significant difference between the index of probability of fall in the elderly with a history of fall and without a history of fall, that is, the elderly without a history of falls performed the test in less time than the elderly with a history of falls $(\mathrm{P}<0.001)$.

\section{Discussion and Conclusion}

This study aimed to evaluate and compare functional balance and the probability of falls in the elderly with a history of falls and no history of falls. The results showed a significant difference between functional balance and the probability of falling in the elderly with a history of falls and without a history of falls. In the elderly with a history of falls, compared to the elderly without a history of falls, there is a more significant balance problem and the possibility of re-falls. Impairment of functional capacity has significant consequences for the family, society, health system, and life of the elderly [23].

The results of this study and other studies show that the elderly with a history of falls have a lower balance score than the elderly without a history of falls; they are also more likely to fall again. As a result, people with a history of falls are more likely to be affected by fear of complexity and are more limited in their daily activities. Given the high prevalence of falls among the elderly, even among independent ones, balance problems must be diagnosed soon; balance problems make older people more susceptible to subsequent falls and possible complications, which may cause limitations and disability. To this end, balance criteria with sufficient sensitivity to elucidate emerging balance disorders seem necessary.

Table 1. Demographic characteristics of the elderly living in nursing homes

\begin{tabular}{ccc}
\hline \multirow{2}{*}{\begin{tabular}{c} 
Memographic Profile \\
\cline { 2 - 3 }
\end{tabular}} & \multicolumn{2}{c}{ Mean \pm SD } \\
\hline The Group With a History of Falls $(\mathbf{n}=\mathbf{1 5})$ & The Group Without a History of Falls $(\mathbf{n}=\mathbf{1 5})$ \\
\hline Height $(\mathrm{cm})$ & $73.5 \pm 3.1$ & $71.6 \pm 7.6$ \\
Mass $(\mathrm{kg})$ & $165.3 \pm 2.5$ & $168.4 \pm 3.1$ \\
\hline
\end{tabular}




\section{Ethical Considerations}

Compliance with ethical guidelines

All ethical principles are considered in this article. The participants were informed of the purpose of the research and its implementation stages. A written consent was obtained from subjects. This study was approved by the Ethics Committee of Guilan University of Medical Sciences (Code: 399.252IR.GUMS.REC).

\section{Funding}

This research did not receive any specific grant from funding agencies in the public, commercial, or not-forprofit sectors.

\section{Authors' contributions}

Data collection and writing - original draft: Seyed Mojtaba Tabatabaiasl and Parisa Sedaghati; Conceptualization, implementation and data analysis: All authors.

\section{Conflicts of interest}

The authors declared no conflict of interest. 


\title{
مقايسه تعادل عملكردى و احتمال سقوط در سالمندان با وبدون سابقه سقوط ساكن در مراكز مراقبتى
}

\author{
سيد مجتبى طباطبايى اصل' (1. "*ريسا صداقتى' نه فريبا جوازى' \\ 1. كروه آسيبشناسى ورزشى وحركات اصلاحى، دانشكده تربيت بدنى و علوم ورزشى، دانشكاه كيلان، رشت، ايران.
}

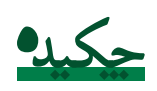

هدف هدف از تحقيق حاضر همقايسه تعادل عملكردى و احتمال سقوط در سالمندان با يا بدون سابقه سقوط ساكن در مراكز مراقبتي بود.

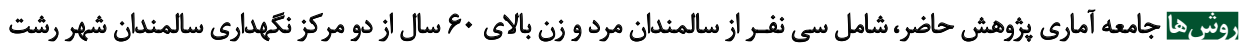

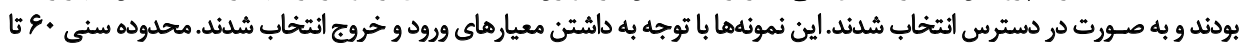

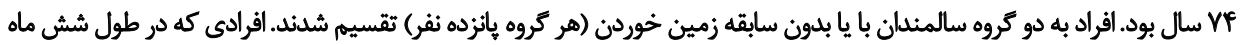

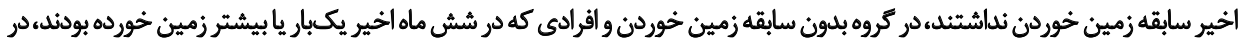

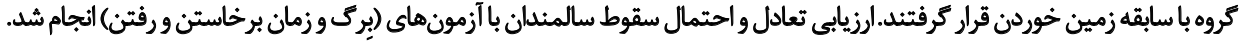

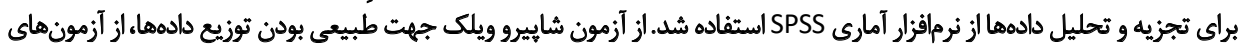

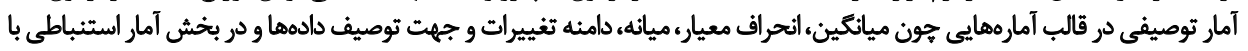

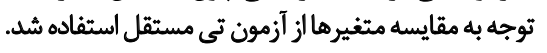

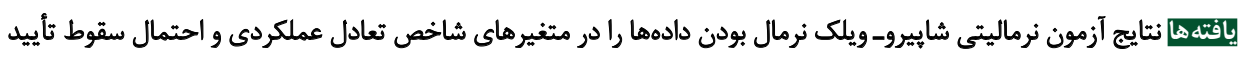

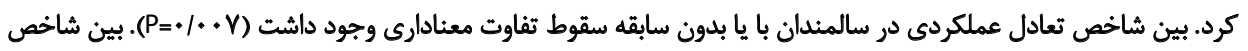

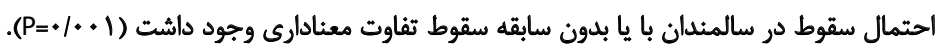

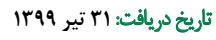
تاريخ يذيرش: ت 19 مرداد

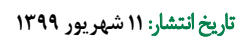

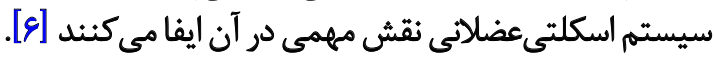

كاهش تعادل در افراد سالمند، همراه با افزايش ميزان زمين

1. Visual

2. Somatosensory

3. Vestibular

dodê

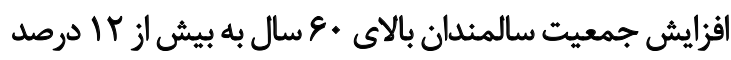

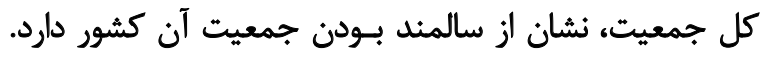

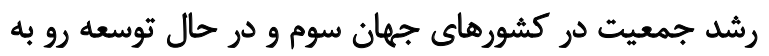

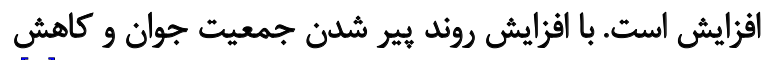

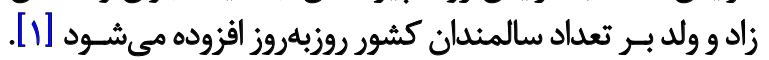

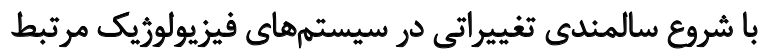

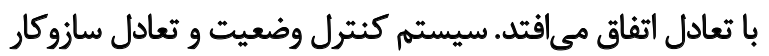

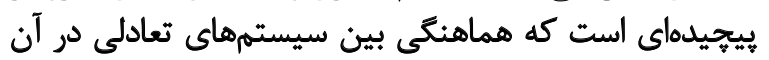
نقش بسزايى دارند [r] سالمندى و بازنشستكى دوره فرسودگى نيست، بلكه دورهاي

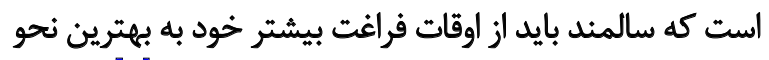

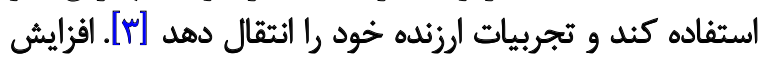


موجود بين سالمندان با يا بدون سابقه سقوط در ايجاد سقوطهاى

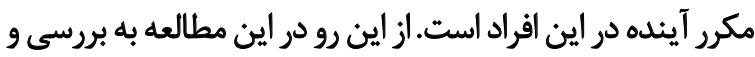

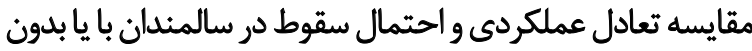
سابقه سقوط يرداخته خُواهد شد.

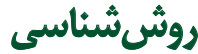

تحقيق حاضر از نوع مطالعات علّى مقايسهايى بود كه به شكل

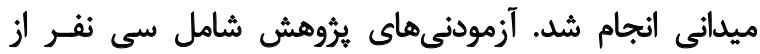

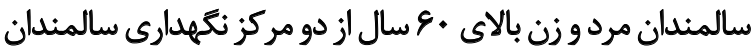
شهر رشت بودند و به صـورت تصادفى و در دسترس انتخ انتخاب

اين نمونهها با توجه به داشتن معيارهاى ورود و خروج انتخاب

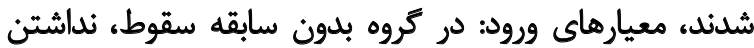

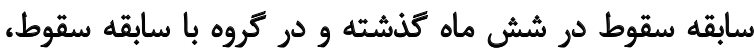

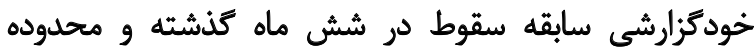

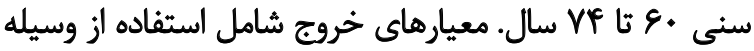

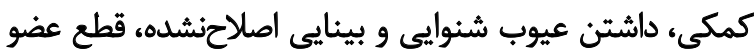

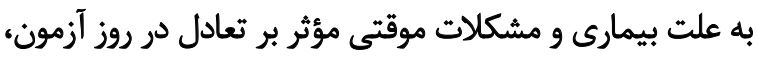

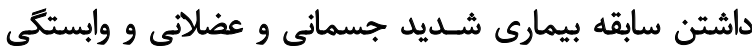

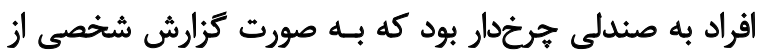

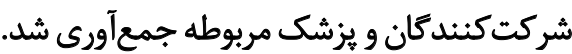

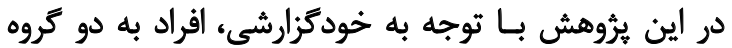

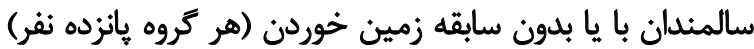

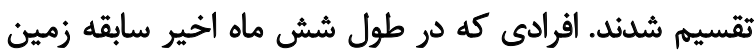

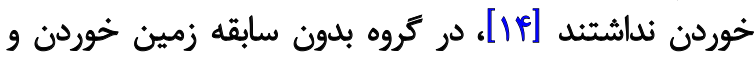

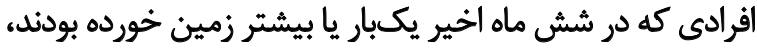

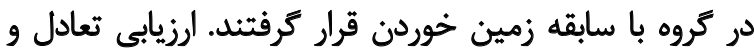

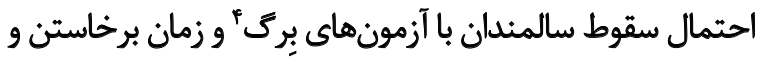

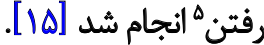

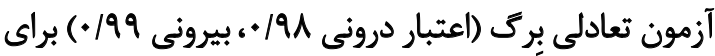

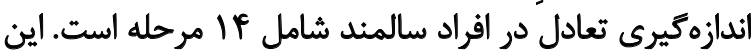

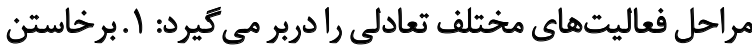

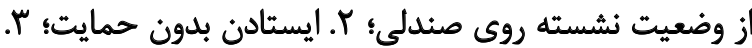

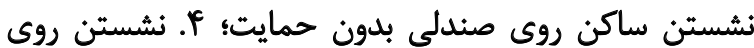

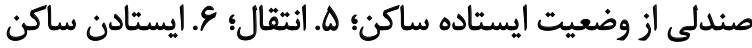

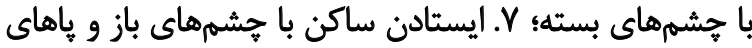

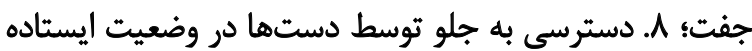

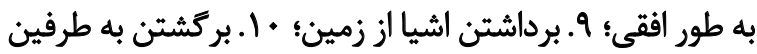

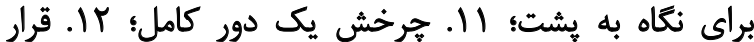

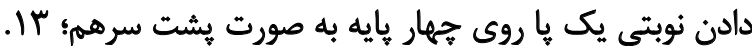

4. Berg test

5. Tug test
خوردن اين افراد است، به طورى كه هر ساله يكسوم سالمندان

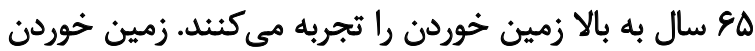

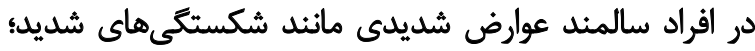

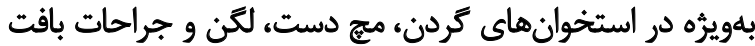

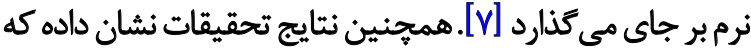

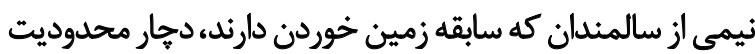

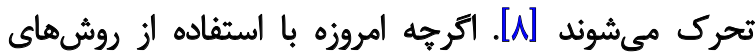

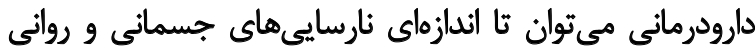

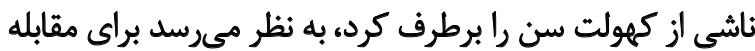

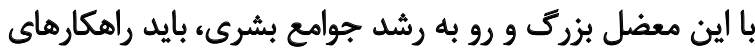

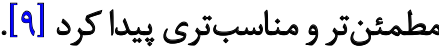

در همين راستا، روشهاى متعددى براى سـنجش تعـادل

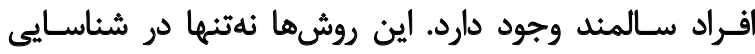

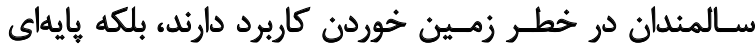

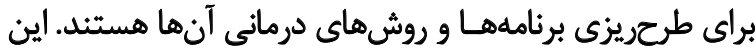

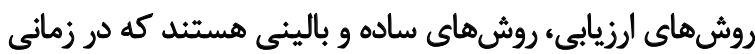

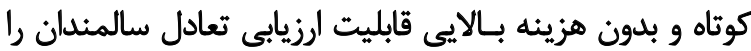

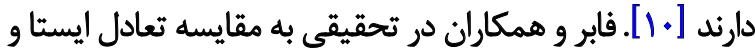

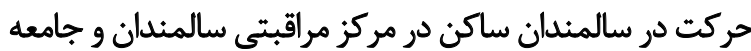

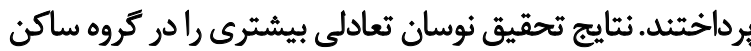

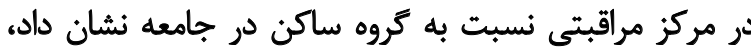

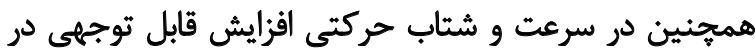

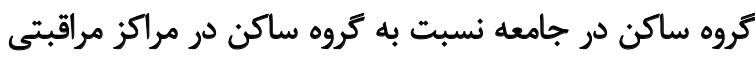

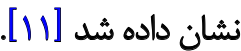

شهربانيان و همكاران در تحقيقى به بررسى مقايسه اثر بر باني

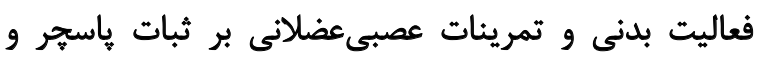

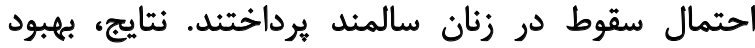

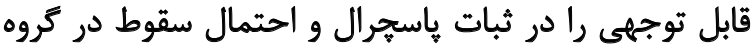

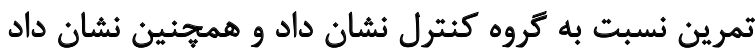

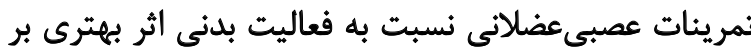
احتمال سقوط در سالمئدان زن دارد [1/]].

ساميا و همكاران در تحقيقى به بررسى مقايسه تعادل

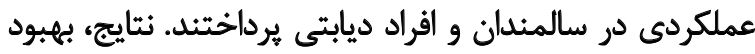

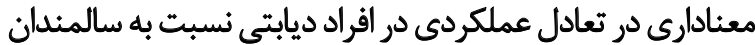

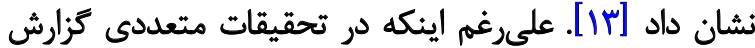

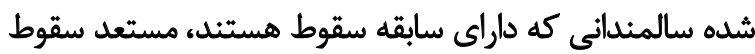
مكررند، ولى در بيشتر تحقيقات به مقايسه تعادل در مرداني

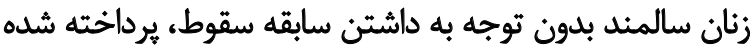

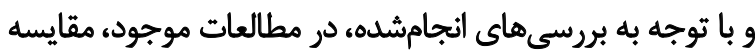

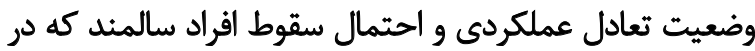

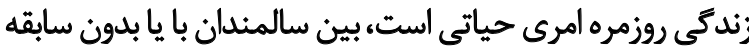
سقوط انجام نشيله است. لزوم انجام اين تحقيق به دليل اهميت بيش آكهى وتفاوتهاى 
جدول ا. مشخصات جمعيتشناختى سالمندان ساكن در مراكز سالميدان

\begin{tabular}{|c|c|c|}
\hline \multicolumn{2}{|c|}{ ميانكين 土|نحراف معيار } & \multirow{2}{*}{ مشخصات جمعين شناختى } \\
\hline كروه بدون سابقه سقوط (N=15) & Sروه با سابقه سقوط (N=15) & \\
\hline$V / P \pm V / 8$ & $n / \Delta \pm r / 1$ & سن (تقويمى) \\
\hline$|\& N F \pm r /|$ & $18 \Delta / r \pm r / \Delta$ & قد (سانتىمتر) \\
\hline $8 \& / A \pm F / 1$ & $R \in / f \pm Y / a$ & وزن (كيلوكرم) \\
\hline
\end{tabular}

مجله بيومكانيك وزنش

جدول r. نتايج آزمون تفاوت شاخص تعادل عملكردى بين كروههاى سالمثدان با يا بدون سابقه سقوط

\begin{tabular}{|c|c|c|c|}
\hline $\mathbf{P}$ & $\mathbf{t}$ & ميانكين ذ انحراف معيار & كروه \\
\hline $.1 . . \mathrm{V}$ & r/ar & $r \Psi / r \pm F / A r$ & سالمثلن با سابقه سقوط \\
\hline & & $r \cdot \pm V / r$ & سالمنان بلدون سابقه سقوط \\
\hline
\end{tabular}

مجله بيومكانيك وزنش

جدول r. نتايج آزمون تفاوت شاخص احتمال سقوط در سالمندان با يا بدون سابقه سقوط

\begin{tabular}{|c|c|c|c|}
\hline $\mathbf{P}$ & $\mathbf{t}$ & 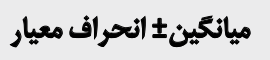 & كروه \\
\hline $.1 .+1$ & $r / \Delta \Delta$ & $r / T \pm r / A$ & سالمنلان با سابقه سقوط \\
\hline & & $\mid V / P \pm \varphi / \varphi$ & سالمندان بلون سابقه سقوط \\
\hline
\end{tabular}

مجله بيومكانيك ورنش

از آزمونهاي آمار توصيفي در قالب آمارمهايي جهون ميانغين،

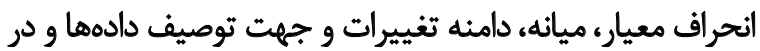

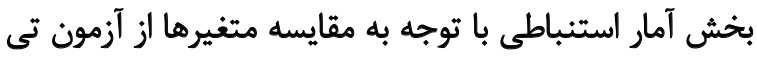
مستقل استفاده شد.

جن

نتايج آزمون نرماليتى شاييرو ويلك، نرمال بودن دادهها رادر

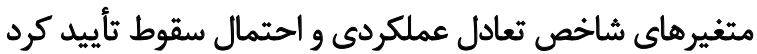
و نتايج مربوط به اطلاعات جمعيتشناختي و مقايسه كروهولى عماي

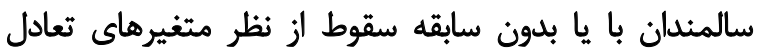

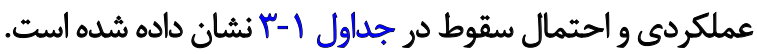

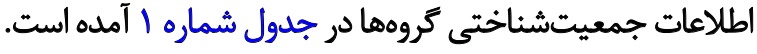
طبق جدول شماره T، با توجه به نتايج آزمون تي مستقل، بين

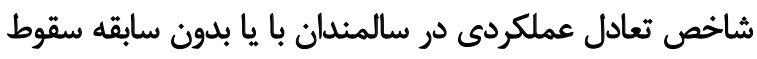

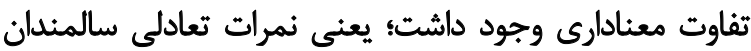

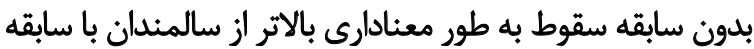

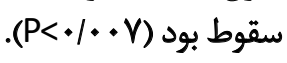

طبق جدول شماره با، با توجه به نتايج آزمون ثي مستقل، بين

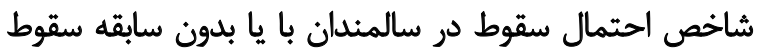

ايستادن در وضعيتي كه يك يا در جلوى باي ديكر قرار دارد؛

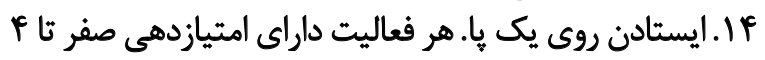

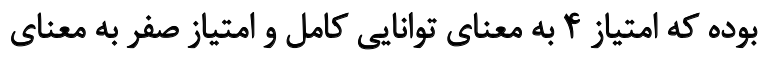

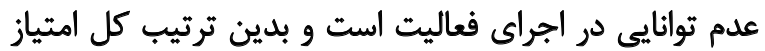

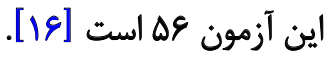
آزمون زمان برخاستن و حركت كردن (يايايي 199٪)، شامل شش مرحله يشت سر هم است. براى انجام، ابتدا يك صند صندلى

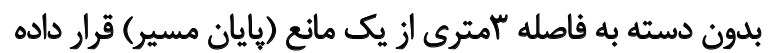

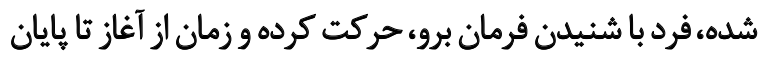

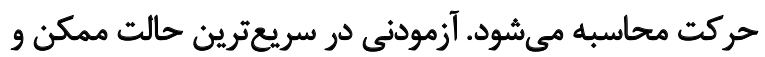

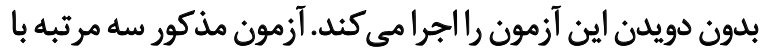

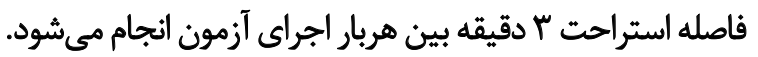

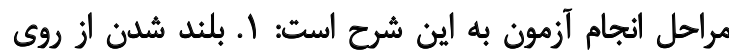

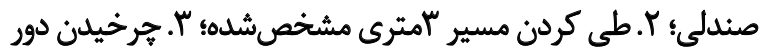

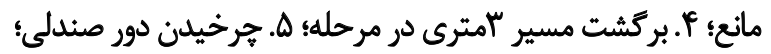

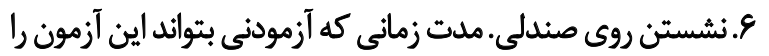

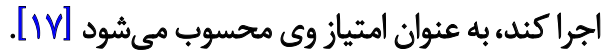
براى تجزيه و تحليل دادهها از نرمافزار آمارى SPSS استفاده

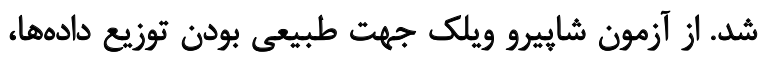


اين روشهاى ارزيابى، روشهايي ساده و بالينى اند كه در زمانى

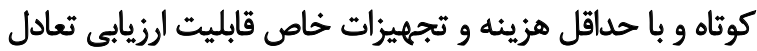

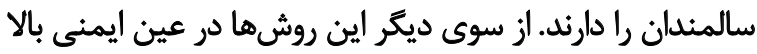

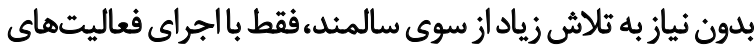

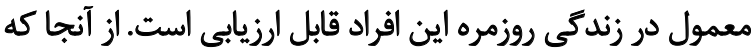

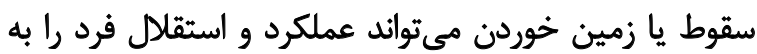

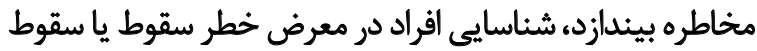

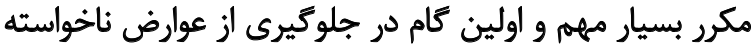

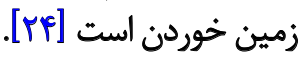

تعيين علل سقوط سالمندان و توسعه روشهاى تشخيصى

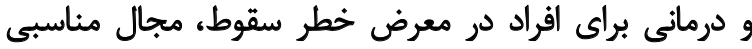

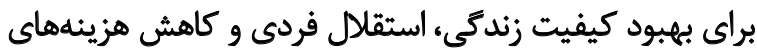

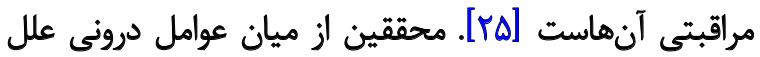

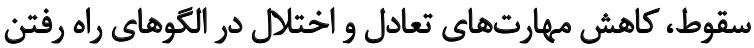

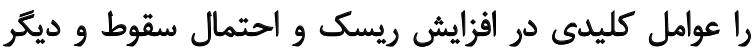

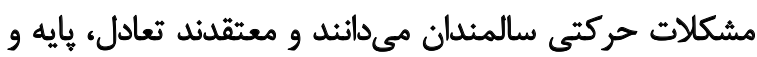

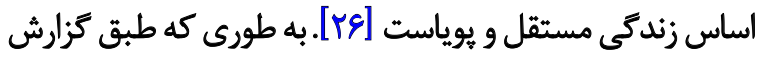

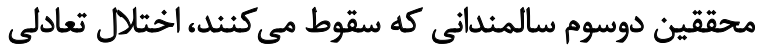

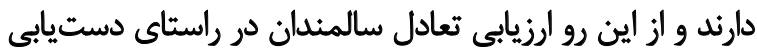

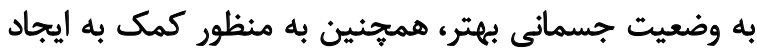

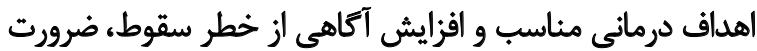

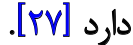

نتايج تحقيق حاضر و ساير تحقيقات انجامشده، نشانكَر اين

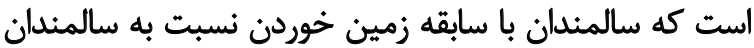

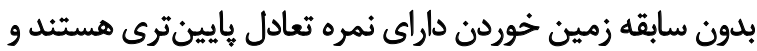

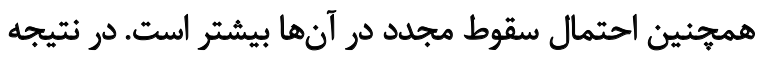

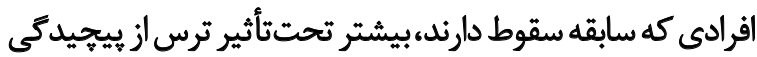

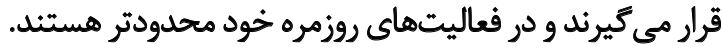
با توجه به شيوع بالاي زمين خوردن در ميان سالمندان، حتى ئى

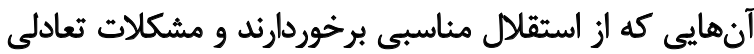

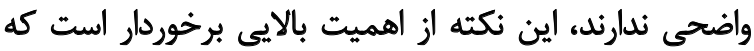

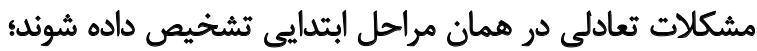

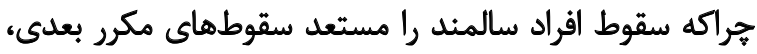

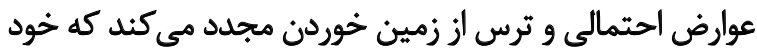

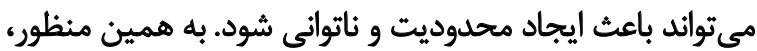

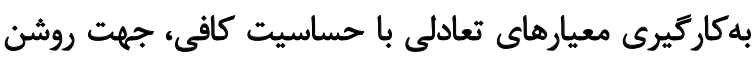

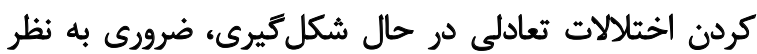

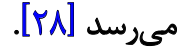

\section{نتيجليَيرىنهايى}

در راستاى نتايج مطالعه حاضر و با توجه به كاهش تعادل

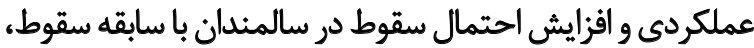

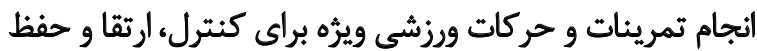

تفاوت معنادارى وجود داشت؛ يعنى سالمندان بدون سابقه سقوط

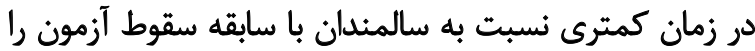

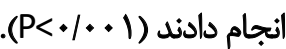

هدف از يروهش حاضر بررسى و مقايسه تعادل عملكردى و

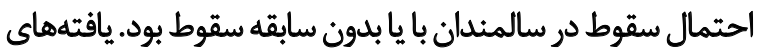

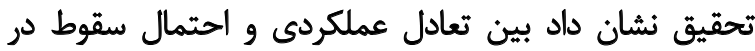

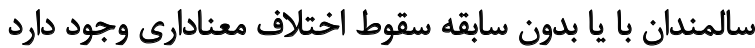

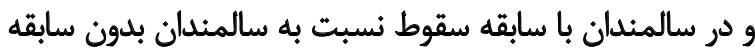

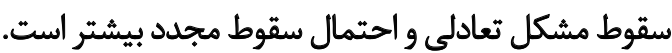

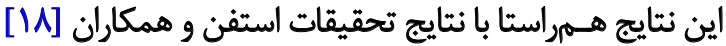

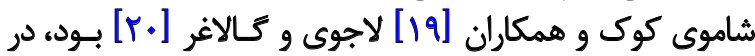

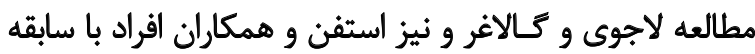

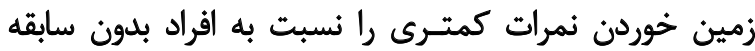

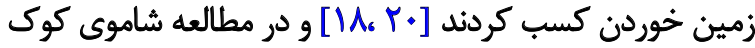

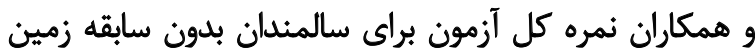

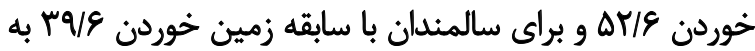

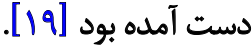

مارتينس و همكاران در تحقيقى به مقايسه احتمال سقوط در

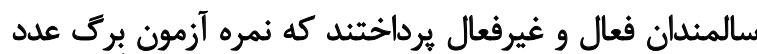

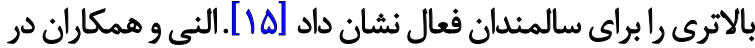

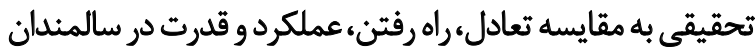

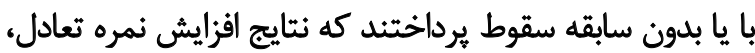

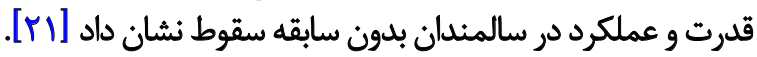
فرايند سالمندى با از دست دادن ظرفيت عملكردي كه باعث

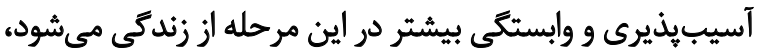

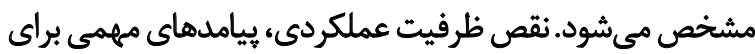

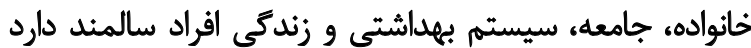

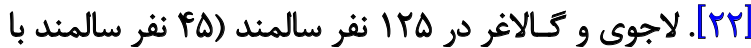

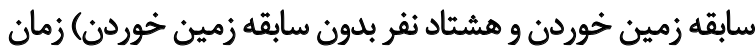

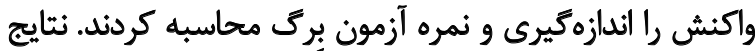

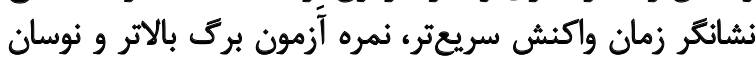

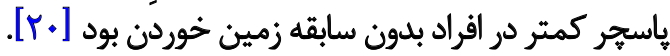
فرايند سالمندى، با از دست دادن ظرفيت عملكردى كه باعث

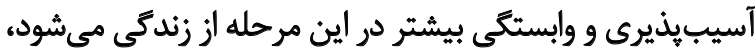

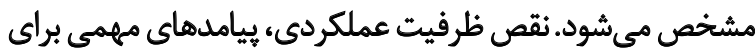

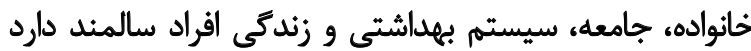

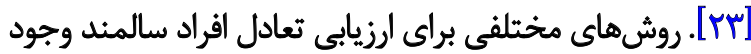

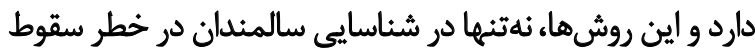

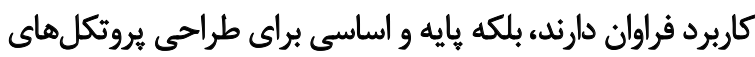

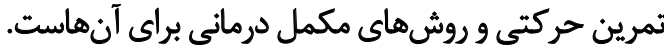




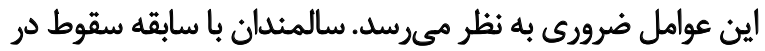

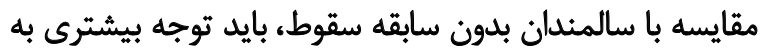
عوامل تعادل و انعطاف يذيرى داشئ داشته باشند.

شركت منظم در فعاليت جسمانى براي به دست آوردن

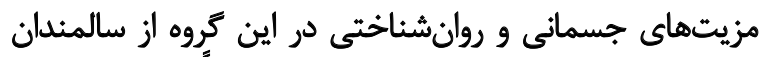

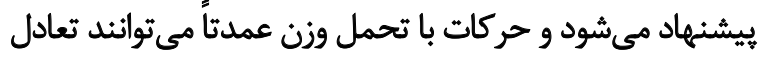

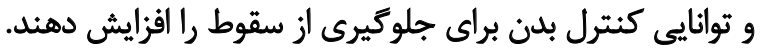

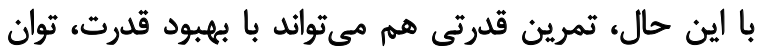

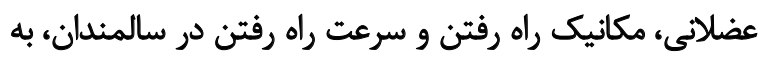

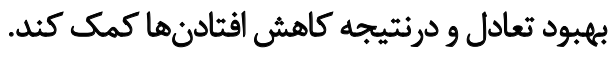

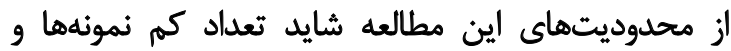

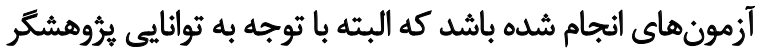

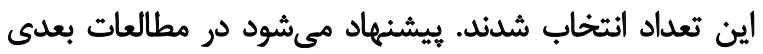

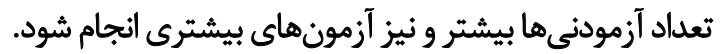

مالاحظات اخلاقي

يبيروى از اصول اخلاق يثوهش

اين مقاله داراى كد اخلاق از كميته اخلاق دانشعاه علوميزشكى

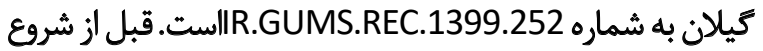

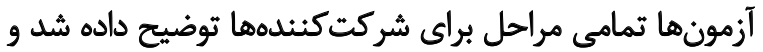

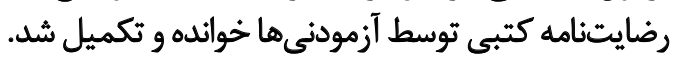

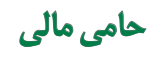

اين يزوهش هيجكونه كمك مالى از سازمانيهاى دولتى،

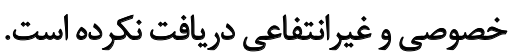

$$
\text { مشاركت ثويسند انَان }
$$

تحليل، نكارش و ويراستارى: سيد مجتبى طباطبايياصل و و

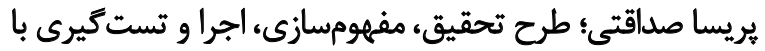
مشاركت همه نويسندكان اين اثر بوده است. مفرمبازئ

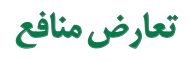

طبق نظر نويسندكان، اين يُؤوهش تعارض منافع ندارد. 


\section{References:}

[1] Ruissen GR, Liu Y, Schmader T, Lubans DR, Harden SM, Wolf SA, et al. Effects of group-based exercise on flourishing and stigma consciousness among older adults: Findings from a randomised controlled trial. Appl Psychol Health Well-Being. 2020; 12(2):559-83. [DOI:10.1111/ aphw.12197] [PMID]

[2] Goudarz M, Foroughan M, Makarem A, Rashedi V. [Relationship between social support and subjective well-being in olde adults (Persian)]. Salmand. 2015; 10(3):110-9. http://salmandj.uswr.ac.ir/article1-953-en.html

[3] Minasian V, Marandi SM, Mojtahedi H, Ghasemi Gh. [The evaluation of health-related physical fitness status of men aged between 50 and 65 in Isfahan and comparison with available norms (Persian)]. J Sports Sci. 2012; 4(14):111-27. [DOI:10.22059/JSB.2013.29531]

[4] Filar-Mierzwa K, Długosz-Boś M, Marchewka A, Aleksander-Szymanowicz P. Effect of different forms of physical activity on balance in older women. J Women Aging. 2020:1-6 [DOI:10.1080/08952841.2020.1718 579] [PMID]

[5] Nickens $\mathrm{H}$. Intrinsic factors in falling among the elderly.Arch Intern Med. 1985; 145(6):1089-93. [DOI:10.1001/archinte.145.6.1089] [PMID]

[6] Seyedjafari E, Sahebozamani M, Ebrahimipour E. [Effect of eight weeks of water exercises on deep part of the pool on the static balance of the elderly man (Persian)]. ISalmand. 2017 Dec 10;12(3):384-93 [DOI:10.21859/sija.12.3.384]

[7] Farsi A, Ashayeri $\mathrm{H}$, Mohammadzadeh S. [The effect of six weeks balance training program on kinematic of walking in women elderly people (Persian)]. Salmand. 2015; 9(4):278-87. http://salmandj.uswr.ac.ir/ article-1-685-en.html

[8] Akbari Kamrani A A, Azadi F, Akbari Kamrani M, Pezhmanfard P, Siyadat $\mathrm{S}$. [The effect of romberg exercise on falling episodes of elderlies with history of past fallings (Persian)]. Iran J Ageing. 2007; 2(2):246-251. http://salmandj.uswr.ac.ir/article-1-2-en.html

[9] Attix DA, Welsh Bohmer KA. Geriatric Neuropsychology: Assessment and Intervention. In: Attix DK, Welsh Bohmer KA, editors. Geriatric Neuropsychology: Assessment and Intervention. New York: Guilford Press; 2006. https://academic.oup.com/acn/article/21/4/367/2919?login=true

[10] Arghavani H, Zolaktaf V, Lenjannejadian S. [Comparing the effects of anticipatory postural adjustments focused training and balance training on postural preparation, balance confidence and quality of life in elderly with history of a fall (Persian)]. Aging Clin Exp Res. 2019 Oct 14:1-9. [DOI:10.1007/s40520-019-01358-5] [PMID]

[11] Faber LM, Moreira LA, Scheicher ME. Comparison of static balance and mobility between nursing home residents and community-dwelling elderly. Manual Ther, Posturology Rehabil J. 2020; 1-7. [DOI:10.17784/ mtprehabjournal.2019.17.749]

[12] Shahrbanian S, Hashemi A, Hemayattalab R. [The comparison of the effects of physical activity and neurofeedback training on postural stability and risk of fall in elderly women: A single-blind randomized controlled trial (Persian)]. Physiother Theory Pract. 2019:1-8. [DOI:10.1080/ 09593985.2019.1630877] [PMID]

[13] Khan S, Amjad I, Tariq H. [Comparison of balance performance between diabetic and elderly population (Persian)]. Int J Rehab Sci (IJRS). 2018; 7(1):12-5. http://research.riphah.edu.pk/id/eprint/742/

[14] Melzer I, Benjuya N, Kaplanski J. Postural stability in the elderly: A comparison between fallers and non-fallers. Age Ageing. 2004; 33(6):602-7. [DOI:10.1093/ageing/afh218] [PMID]
[15] Franciulli PM, Souza PA, Soares PN, Silva VN, Severino YT, Dos Santos YG, et al. Comparison of the risk of falls between elderly people who practice physical exercises and who are sedentary and the relationship between balance and muscle strength variables. Mundo da Saude. 2019; 43(2):360-73. [DOI:10.15343/0104-7809.20194302360373]

[16] Kashani V, Zarifkar M, Alinaghipoor Z. [Determining validity and reliability of the Persian version of Activities-specific Balance Confidence Scale for elderly (Persian)]. Koomesh. 2018; 20(4):Pe705-Pe712. https:// www.cabdirect.org/cabdirect/abstract/20193073915

[17] Asadi Samani Z, Rahnama N, Reisi J, Lenjan Nejadian S. [Correlation between new activity-based balance index with accelerometer data and postural balance in elderly woman (Persian)]. Koomesh. 2020; 22(1):928. [DOI:10.29252/koomesh.22.1.92]

[18] Steffen TM, Hacker TA, Mollinger L. Age- and gender- related test performance in community-dwelling elderly people: Six minute walk test berg balance scale, timed up \& go test, and gait speeds. Phys Ther. 2002 88(2):128-137. [DOI:10.1093/ptj/82.2.128] [PMID]

[19] Shumway-Cook A, Baldwin M, Polissar NL, Grubar W. Predicting the probability for falls in community-dwelling older adults. Phys Ther. 1997; 77(8):812-819. [DOI:10.1093/ptj/77.8.812] [PMID]

[20] Lajoie Y, Gallagher SP. Predicting falls within the elderly community: comparison of postural sway, reaction time, the berg balance scale and the Activities-Specific Balance Confidence (ABC) scale for comparing fallers and non-fallers. Arch Gerontol Geriatr. 2004; 38(1):11-26. [DOI:10.1016/S0167-4943(03)00082-7]

[21] Cebolla EC, Rodacki AL, Bento PC. Balance, gait, functionality and strength: Comparison between elderly fallers and non-fallers. Braz J Phys Ther. 2015; 19(2):146-51. [DOI:10.1590/bjpt-rbf.2014.0085] [PMID] [PMCID]

[22] Maeshiro FL, Lopes MC, Okuno MF, Camapanharo CR, Batista RE. Capacidade funcional e a gravidade do trauma em idosos. Acta Paulista de Enfermagem. 2013; 26(4):389-94. [DOI:10.1590/S010321002013000400014]

[23] Abdoli B, Shams A, Shamsipour Dehkordi P. [The effect of practice type on static and dynamic balance in elderly 60-75 year old women with no history of falling (Persian)]. Daneshvar. 2012; 19(97):43-50. http:// daneshvarmed.shahed.ac.ir/article_1501_5af6ef95403f1637bf70e78cf f42382d.pdf

[24] Satterfied KS. Balance testing helps identify elderly at risk of multiple falls. Am Otol Soc. 2001 May. Link No Found.

[25] Shumway-Cook A, Brauer S, Woollacott M. Predicting the probability for falls in community-dwelling older adults using the Timed Up \& Go Test. Physical Therapy. 2000; 80(9):896-903. [DOI:10.1093/ptj/80.9.896] [PMID]

[26] Shumway-Cook A, Woollacott MH. Motor control: Translating research into clinical practice. Philadelphia: Lippincott Williams \& Wilkins; 2007. https://books.google.com.sa/books?id=BJcL3enz3xMC\&printsec $=$ frontcover $\& d q=$

[27] Woollacott M, Shumway-Cook A. Attention and the control of posture and gait: a review of an emerging area of research. Gait Posture. 2002 16(1):1-4. [DOI:10.1016/S0966-6362(01)00156-4]

[28] Daly RM, Rosengren BE, Alwis G, Ahlborg HG, Sernbo I, Karlsson MK. Gender specific age-related changes in bone density, muscle strength and functional performance in the elderly: A-10 year prospective population-based study. BMC Geriatr. 2013; 13(1):1-9.[DOI:10.1186/14712318-13-71] [PMID] [PMCID] 
This Page Intentionally Left Blank 